\title{
Asymptotic Performance Analysis of V-BLAST *
}

\author{
Yi Jiang ${ }^{\dagger}$ Xiayu Zheng Jian Li
}

\begin{abstract}
In this paper, we present an asymptotic analysis of the V-BLAST scheme at high signal-to-noise ratio (SNR) region. We consider point-to-point MIMO communications over an i.i.d. Rayleigh flat fading channel with $n$ transmitting antennas and $m(m \geq n)$ receiving antennas. Both the zero-forcing V-BLAST (ZF-V-BLAST) and minimum mean-squared-error V-BLAST (MMSE-VBLAST) are analyzed with respect to their diversity gains and BER performances. We show that the diversity gain of V-BLAST, including ZF-V-BLAST and MMSE-V-BLAST, with optimal ordering is $m-n+1$. I.e., applying the optimal ordering technique does not improve the diversity gain. Contrary to the common perception that the MMSE and ZF estimators have asymptotically the same post-processing SNR for high input SNR, we show that the difference between the post-processing SNRs of the two estimators does not vanish for high SNR. We also quantify the remarkable BER performance advantage of the MMSE-V-BLAST over the ZF-V-BLAST for high SNR.
\end{abstract}

\section{INTRODUCTION}

It is well-known that deploying multiple antennas at both the transmitter and receiver sides can drastically improve the channel capacity. Many schemes have been proposed to exploit the high spectral efficiency of MIMO channels, among which V-BLAST is relatively simple to implement and can reap a large portion of the high spectral efficiency. At the transmitter, V-BLAST de-multiplexes the input data streams into $n$ independent substreams, which are transmitted in parallel over the $n$ transmitting antennas. At the receiver end, the antennas receive the substreams, which are mixed and superimposed by noise. By applying sequential interference nulling and cancellation, the receiver can separate the substreams one by one [1]. Although V-BLAST is known to be equivalent to a decision feedback equalizer and is optimal in terms of achieving the channel capacity [2] [3], it suffers from poor diversity gain. In an i.i.d. Rayleigh flat fading channel with $n$ transmitting antennas and $m$ receiving antennas $(m \geq n)$, the first detected substream has a diversity gain of only $m-n+1$. Due to the error propagation effect, the detection error of the first substream can result in more errors to the subsequent substreams detection. Hence the first substream is the bottleneck which limits the overall performance of the scheme. One can apply the optimal ordering technique to mitigate this bottleneck effect [1]. That is, at each step one should detect the data substream with the largest post-processing SNR. It is shown in [4] that the optimal ordering does not improve the diversity

\footnotetext{
${ }^{*}$ This work was supported in part by the National Science Foundation Grant CCR-0104887.

${ }^{\dagger}$ Yi Jiang, Xiayu Zheng, and Jian Li are with the Department of Electrical and Computer Engineering, University of Florida, Gainesville, FL 32611-6130, USA. Email: \{yjiang, li\}@dsp.ufl.edu, xiayueufl.edu
}

gain when there are two transmitting antennas $(n=2)$. However, it remains unknown if applying optimal ordering can help improve the diversity gain in general cases. Moreover, almost all the existing performance analyses are confined to ZF-VBLAST. In this paper, we show that for both ZF-V-BLAST and MMSE-V-BLAST, using optimal ordering does not help improve the diversity gain, which remains at $m-n+1$. We also show that the difference between the post-processing SNRs of MMSE-V-BLAST and ZF-V-BLAST converges to a scaled $\mathcal{F}$-distribution for high input SNR. Contrary to the common perception that the MMSE and ZF estimators have asymptotically the same post-processing SNR for high input SNR, we show that the difference between the post-processing SNRs of the two estimators does not vanish for high SNR. We also quantify the remarkable BER performance advantage of the MMSE-V-BLAST over the ZF-V-BLAST.

\section{Channel Model and Preliminaries}

\section{A. Channel Model}

We consider a communication system with $n$ transmitting and $m$ receiving antennas in an i.i.d. Rayleigh frequency flat fading channel. The sampled baseband signal is given by

$$
\mathbf{y}=\mathbf{H x}+\mathbf{z},
$$

where $\mathbf{y} \in \mathbb{C}^{m \times 1}$ is the received signal and $\mathbf{H} \in \mathbb{C}^{m \times n}$ is the Rayleigh flat fading channel. Throughout this paper, we assume that the entries of $\mathbf{H}$ are i.i.d. and circularly symmetric Gaussian random variables with zero-mean and unit variance, i.e., $h_{i j} \sim N(0,1)$ for $1 \leq i \leq m, 1 \leq j \leq n$. We also assume that the transmitted substreams are independent and have uniform power, i.e., $\mathbf{x} \in \mathbb{C}^{n \times 1}$ has covariance matrix $E\left[\mathbf{x x}^{*}\right]=\sigma_{x}^{2} \mathbf{I}$, where $E[\cdot]$ stands for the expected value and $(\cdot)^{*}$ is the conjugate transpose. The noise $\mathbf{z} \sim N\left(0, \sigma_{z}^{2} \mathbf{I}\right)$ is also circularly symmetric complex Gaussian. We define the input SNR to be

$$
\mathrm{snr}=\frac{\sigma_{x}^{2}}{\sigma_{z}^{2}}
$$

Denoting $\mathbf{h}_{i} \in \mathbb{C}^{m \times 1}$ to be the $i$ th $(1 \leq i \leq n)$ column of $\mathbf{H}$, we can rewrite (1) as

$$
\mathbf{y}=\sum_{i=1}^{n} \mathbf{h}_{i} x_{i}+\mathbf{z}
$$

To separate the transmitted substreams at the receiver, VBLAST first estimates $x_{n}$, which we also refer to as the $n$th layer, and then cancels it out from the received signal vector. Next, it estimates the signal $x_{n-1}$, and so on. The signal estimator can be either the ZF or MMSE estimator, corresponding to ZF-V-BLAST and MMSE-V-BLAST, respectively. To estimate $x_{n}$, the receiver needs to null out $n-1$ interferences from the directions of $\mathbf{h}_{1}, \ldots, \mathbf{h}_{n-1}$, which consumes $n-1$ degrees 
of freedom. Assuming correct detection of $x_{n}$, the signal component $\mathbf{h}_{n} x_{n}$ is subtracted out from $\mathbf{y}$ before the next step of detection. Hence the estimation of $x_{n-1}$ only needs to null out the remaining $n-2$ interferences. Intuitively, the detection of $x_{n-1}$ is more reliable than that of $x_{n}$, and so on.

To measure the reliability of a communication scheme, we refer to the concept of diversity gain [5].

Definition 1 Let $P_{e}(\mathrm{snr})$ denote the average error probability of a scheme with snr. The diversity gain of the scheme is

$$
d=-\lim _{\mathrm{snr} \rightarrow \infty} \frac{\log P_{e}(\mathrm{snr})}{\log \mathrm{snr}} .
$$

The diversity gain measures how fast the error probability decays with SNR. It is known that for the ZF-V-BLAST scheme applied to (1), the $i$ th detected substream has a diversity gain of $m-n+i$, for $1 \leq i \leq n$ (see, e.g., [6], [4]). Equivalently, the $i$ th detected substream has BER

$$
P_{e, i}(\mathrm{snr}) \propto \mathrm{snr}^{-(m-i+1)}, \quad 1 \leq i \leq n .
$$

Clearly, at high SNR, the overall BER of ZF-V-BLAST is dominated by the detection error of the $n$th layer. Moreover, the detection error of the first substream (the $n$th layer) can seriously influence the subsequent substreams. Based on these observations, we focus on analyzing the $n$th layer, since it yields most information on the performance of the V-BLAST scheme.

\section{B. Preliminaries}

We provides two useful theorems as follows. The first theorem is can be found in [7, Lemma 2.1].

Theorem II.1 Let $\mathbf{H}$ be an $m \times n$ complex Gaussian matrix, whose entries are i.i.d. complex Gaussian random variables with zero-mean and unit variance. Denote its $Q R$ decomposition by $\mathbf{H}=\mathbf{Q R}$. The matrix $\mathbf{R}$ is upper triangular with realvalued diagonal. The entries of $\mathbf{R}$ are independent of each other. Moreover, the square of the ith diagonal element of $\mathbf{R}$, $r_{i i}^{2}$, is of chi-square distribution ${ }^{1}$ with the degree of freedom $2(m-i+1)$, which we denote as $\chi_{2(m-i+1)}^{2}$. The off diagonal elements $r_{i j}$, for $1 \leq i<j \leq n$, are zero-mean complex Gaussian with unit variance.

The second theorem is a direct subsequent of Weyl's theorem [8].

Theorem II.2 For an upper triangular matrix $\mathbf{R} \in \mathbb{C}^{n \times n}$, the smallest singular value

$$
\sigma_{\min }(\mathbf{R}) \leq \min _{1 \leq i \leq n}\left|r_{i i}\right|
$$

where $\left\{r_{i i}\right\}_{i=1}^{n}$ form the diagonal of $\mathbf{R}$.

\section{ANALYSIS OF ZF-V-BLAST}

The ZF-V-BLAST scheme can be represented by the QR decomposition $\mathbf{H}=\mathbf{Q R}$, where $\mathbf{R}$ is an $n \times n$ upper triangular

\footnotetext{
${ }^{1}$ Throughout of this paper, the chi-square distribution with the degree of freedom $l$ is defined as the sum of the square of $l$ independent real-valued zero-mean Gaussian variable with variance $1 / 2$. It is slightly different from the standard chi-square distribution
}

matrix and $\mathbf{Q}$ is an $m \times n$ matrix with its orthonormal columns being the ZF nulling vectors. Let us rewrite (1) as

$$
\mathbf{y}=\mathbf{Q R x}+\mathbf{z}
$$

Multiplying $\mathbf{Q}^{*}$ to both sides of (6) yields

$$
\tilde{\mathbf{y}}=\mathbf{R x}+\tilde{\mathbf{z}}
$$

or

$$
\left[\begin{array}{c}
\tilde{y}_{1} \\
\tilde{y}_{2} \\
\vdots \\
\tilde{y}_{n}
\end{array}\right]=\left[\begin{array}{cccc}
r_{11} & r_{12} & \ldots & r_{1 n} \\
0 & r_{22} & \ldots & r_{2 n} \\
\vdots & \ddots & \ddots & \vdots \\
0 & \ldots & 0 & r_{n n}
\end{array}\right]\left[\begin{array}{c}
x_{1} \\
x_{2} \\
\vdots \\
x_{n}
\end{array}\right]+\left[\begin{array}{c}
\tilde{z}_{1} \\
\tilde{z}_{2} \\
\vdots \\
\tilde{z}_{n}
\end{array}\right]
$$

The sequential signal detection is as follows

$$
\begin{aligned}
& \text { for } i=n:-1: 1 \\
& \quad \hat{x}_{i}=\mathcal{C}\left[\left(\tilde{y}_{i}-\sum_{j=i+1}^{n} r_{i j} \hat{x}_{j}\right) / r_{i i}\right]
\end{aligned}
$$

end

where $\mathcal{C}$ stands for mapping to the nearest symbol in the symbol constellation. For simplicity, ignoring the propagation error effect, we can regard the resulting layers, which correspond to different substreams, as

$$
y_{i}=r_{i i} x_{i}+z_{i}, \quad \text { for } \quad i=1, \ldots, n \text {. }
$$

where $r_{i i}^{2} \sim \chi_{2(m-i+1)}^{2}$, or,

$$
f_{r_{i i}^{2}}(x)=\frac{1}{(m-i) !} x^{m-i} e^{-x} .
$$

The post-processing SNR is

$$
\rho_{i}^{\mathrm{ZF}}=r_{i i}^{2} \mathrm{snr} .
$$

Recall that the diversity gain is defined as

$$
d=-\lim _{\mathrm{snr} \rightarrow \infty} \frac{\log P_{e}(\mathrm{snr})}{\log \mathrm{snr}} .
$$

A direct calculation of (12) is often difficult. However, one can apply the typical error event analysis technique to derive the diversity gain (see [6, Ch. 3]). The typical error event is defined as

$$
\mathcal{E}_{i}=\left\{\mathbf{H}: r_{i i}^{2}<\mathrm{snr}^{-1}\right\}
$$

It can be shown that the diversity gain of the $i$ th layer is (see e.g., [6])

$$
d_{i}=-\lim _{\mathrm{snr} \rightarrow \infty} \frac{\log P\left(\mathcal{E}_{i}\right)}{\log \mathrm{snr}}=m-i+1 .
$$

We see that the larger the $i$ is, the smaller the diversity gain the $i$ th layer has. Consequently, the largest or $n$th layer, limits the overall performance of ZF-V-BLAST at high SNR.

To improve the BER performance of the $n$th layer, one can apply the optimal detection ordering technique. For each realization of $\mathbf{H}$, instead of fixing the $n$th layer as the data corresponding to $\mathbf{h}_{n}$, one can permute the columns of $\mathbf{H}$ such that the channel gain of the $n$th layer, $r_{n n}$, is maximized as proposed in [1]. The reordering technique can significantly improve the BER performance. In [4], the special case of $n=2$ 
is analyzed and it is shown that the reordering technique does not improve the diversity gain. This finding coincides with the result presented in [5], where $m-1$ is given as an upper bound of the diversity gain of V-BLAST with optimal ordering. However, the exact diversity gain of the V-BLAST equalizer with optimal ordering was unknown for the general case of $n>2$. We attempt to answer this question in the following.

We focus on the $n$th layer. By permuting the columns of $\mathbf{H}$, one has $n$ options of which substream to be detected first. Let $\mathbf{H}^{(i)}$ denote the matrix obtained by exchanging the $i$ th and $n$th columns of $\mathbf{H}$ (As a special case, $\mathbf{H}^{(n)}=\mathbf{H}$ ). Let $\mathbf{H}^{(i)}=$ $\mathbf{Q}^{(i)} \mathbf{R}^{(i)}$ be the QR decomposition and $r_{n n}^{(i)}$ be the $(n, n)$ th entry of $\mathbf{R}^{(i)}$ (As a special case, $r_{n n}^{(n)}=r_{n n}$ ). According to the typical error event analysis technique, the diversity gain of ZF-V-BLAST with optimal ordering can be calculated as

$$
\begin{aligned}
d_{n, \mathrm{ZF}}^{\text {ord }}=-\lim _{\mathrm{snr} \rightarrow \infty} \frac{\log P\left(\left\{\left|r_{n n}^{(i)}\right|^{2}<\mathrm{snr}^{-1}\right\}_{i=1}^{n}\right)}{\log \mathrm{snr}} \\
=-\lim _{\mathrm{snr} \rightarrow \infty} \frac{\log P\left(\left\{\left|r_{n n}^{(i)}\right|^{2}<\mathrm{snr}^{-1}\right\}_{i=1}^{n-1} \mid r_{n n}^{2}<\mathrm{snr}^{-1}\right)}{\log \mathrm{snr}} \\
+m-n+1 .
\end{aligned}
$$

We now show that the conditional probability

$$
P\left(\left\{\left|r_{n n}^{(i)}\right|^{2}<\operatorname{snr}^{-1}\right\}_{i=1}^{n-1} \mid r_{n n}^{2}<\operatorname{snr}^{-1}\right)
$$

is lower bounded by a strictly positive number unrelated to snr. Rewrite $\mathbf{R}$ as

$$
\mathbf{R}=\left(\begin{array}{ccc}
\times & \ldots & \times \\
0 & r_{i i} & \mathbf{r}_{i}^{*} \\
0 & \mathbf{0} & \mathbf{R}_{i}
\end{array}\right)
$$

where $\mathbf{R}_{i}$ is the tailing $(n-i) \times(n-i)$ upper triangular submatrix of $\mathbf{R}$ and $\mathbf{r}_{i} \in \mathbb{C}^{(n-i) \times 1}$. For notational simplicity, we denote

$$
\overline{\mathbf{R}}_{i}=\left[\begin{array}{c}
\mathbf{r}_{i}^{*} \\
\mathbf{R}_{i}
\end{array}\right] .
$$

Then the last element of $\mathbf{R}^{(i)}, r_{n n}^{(i)}$, satisfies

$$
\begin{aligned}
\left|r_{n n}^{(i)}\right|^{2} & =\left[r_{i i} \vdots \mathbf{0}^{T}\right] \mathbf{P}_{\overline{\mathbf{R}}_{i}}^{\perp}\left[\begin{array}{c}
r_{i i} \\
\mathbf{0}
\end{array}\right] \\
& =\left[r_{i i} \vdots \mathbf{0}^{T}\right]\left(\mathbf{I}-\overline{\mathbf{R}}_{i}\left(\overline{\mathbf{R}}_{i}^{*} \overline{\mathbf{R}}_{i}\right)^{-1} \overline{\mathbf{R}}_{i}^{*}\right)\left[\begin{array}{c}
r_{i i} \\
\mathbf{0}
\end{array}\right] \\
& =r_{i i}^{2}\left(1-\mathbf{r}_{i}^{*}\left(\mathbf{r}_{i} \mathbf{r}_{i}^{*}+\mathbf{R}_{i}^{*} \mathbf{R}_{i}\right)^{-1} \mathbf{r}_{i}\right) \\
& =\frac{r_{i i}^{2}}{1+\mathbf{r}_{i}^{*}\left(\mathbf{R}_{i}^{*} \mathbf{R}_{i}\right)^{-1} \mathbf{r}_{i}} \\
& <\frac{r_{i i}^{2}}{\mathbf{r}_{i}^{*}\left(\mathbf{R}_{i}^{*} \mathbf{R}_{i}\right)^{-1} \mathbf{r}_{i}}
\end{aligned}
$$

In (17), $\mathbf{P}_{\overline{\mathbf{R}}_{i}}^{\perp}$ stands for the orthogonal projection onto the null space of $\overline{\mathbf{R}}_{i}^{T}$. Given $r_{n n}^{2}<\mathrm{snr}^{-1}$, i.e., $r_{n n}<\mathrm{snr}^{-1 / 2}$, it follows from Theorem II. 2 that the smallest singular value of $\mathbf{R}_{i}$ is less than $\mathrm{snr}^{-1 / 2}$. Then the largest singular value of $\left(\mathbf{R}_{i}^{*} \mathbf{R}_{i}\right)^{-1}$ is greater than snr. Hence

$$
\mathbf{r}_{i}^{*}\left(\mathbf{R}_{i}^{*} \mathbf{R}_{i}\right)^{-1} \mathbf{r}_{i}>\operatorname{snrr}_{i}^{*} \mathbf{v v}^{*} \mathbf{r}_{i},
$$

where $\mathbf{v}$ is the eigenvector of $\left(\mathbf{R}_{i}^{*} \mathbf{R}_{i}\right)^{-1}$ corresponding to the largest eigenvalue and $\mathbf{v}^{*} \mathbf{v}=1$. Combining (19) and (18), we obtain that

$$
\left|r_{n n}^{(i)}\right|^{2}<\frac{r_{i i}^{2}}{\left|\mathbf{r}_{i}^{*} \mathbf{v}\right|^{2}} \mathrm{snr}^{-1}
$$

We emphasize that (20) holds as long as $r_{n n}^{2}<\mathrm{snr}^{-1}$ and no matter what the other entries of $\mathbf{R}_{i}$ are. Consequently, we have

$$
\begin{aligned}
& P\left(\left|r_{n n}^{(i)}\right|^{2}<\operatorname{snr}^{-1} \mid\left\{\left|r_{n n}^{(j)}\right|^{2}<\mathrm{snr}^{-1}\right\}_{j=i+1}^{n}\right) \\
> & P\left(\frac{r_{i i}^{2}}{\left|\mathbf{r}_{i}^{*} \mathbf{v}\right|^{2}}<1\right) .
\end{aligned}
$$

Since $\mathbf{r}_{i} \sim N(0, \mathbf{I})$ (cf. Theorem II.1) and $\mathbf{v}^{*} \mathbf{v}=1$, we have

$$
\left|\mathbf{r}_{i}^{*} \mathbf{v}\right|^{2} \sim \chi_{2}^{2}
$$

Note that $r_{i i}^{2} \sim \chi_{2(m-i+1)}^{2}$ and is independent of $\mathbf{r}_{i}$. Hence

$$
\frac{r_{i i}^{2} /(m-i+1)}{\left|\mathbf{r}_{i}^{*} \mathbf{v}\right|^{2}} \sim \mathcal{F}_{2(m-i+1), 2},
$$

and consequently, the lower bound given in (21) is finitely small and is not related to snr. The numerator in (15) can be written as

$$
\begin{aligned}
& P\left(\left\{\left|r_{n n}^{(i)}\right|^{2}<\mathrm{snr}^{-1}\right\}_{i=1}^{n-1} \mid r_{n n}^{2}<\mathrm{snr}^{-1}\right) \\
= & \prod_{i=1}^{n-1} P\left(\left|r_{n n}^{(i)}\right|^{2}<\mathrm{snr}^{-1} \mid\left\{\left|r_{n n}^{(j)}\right|^{2}<\mathrm{snr}^{-1}\right\}_{j=i+1}^{n}\right),
\end{aligned}
$$

which is also a finitely small number. Based on this observation and (15), we have proven the following main result of this section.

Theorem III.1 For the MIMO channel of (1), the diversity gain of ZF-V-BLAST with optimal ordering is

$$
d_{n, Z F}^{\text {ord }}=m-n+1,
$$

i.e., applying optimal ordering does not help improve the diversity gain of $Z F-V-B L A S T$.

\section{ANALYSIS OF MMSE-V-BLAST}

For MMSE-V-BLAST, the nulling vector for the $i$ th layer is [3]

$$
\mathbf{w}_{i}=\left(\mathbf{H}_{i} \mathbf{H}_{i}^{*}+\frac{1}{\mathrm{snr}} \mathbf{I}\right)^{-1} \mathbf{h}_{i}, \quad i=1, \ldots, n,
$$

where $\mathbf{H}_{i} \in \mathbb{C}^{m \times i}$ consists of the first $i$ columns of $\mathbf{H}$. Then the post-processing SNR of the $i$ th layer is

$$
\rho_{i}^{\mathrm{MMSE}}=\frac{\left|\mathbf{h}_{i}^{*} \mathbf{w}_{i}\right|^{2}}{\mathbf{w}_{i}^{*}\left(\mathbf{H}_{i-1} \mathbf{H}_{i-1}^{*}+\operatorname{snr}^{-1} \mathbf{I}\right) \mathbf{w}_{i}} .
$$

Inserting (25) into (26), we can simplify (26) via some straightforward calculations to get (see, e.g., [3])

$$
\rho_{i}^{\mathrm{MMSE}}=\mathbf{h}_{i}^{*} \mathbf{C}_{i}^{-1} \mathbf{h}_{i}, \quad i=1, \ldots, n,
$$


where $\mathbf{C}_{i}=\mathbf{H}_{i-1} \mathbf{H}_{i-1}^{*}+\frac{1}{\mathrm{snr}} \mathbf{I}$. Applying the matrix inversion lemma, we obtain

$$
\mathbf{C}_{i}^{-1}=\operatorname{snr}\left[\mathbf{I}-\mathbf{H}_{i-1}\left(\mathbf{H}_{i-1}^{*} \mathbf{H}_{i-1}+\frac{1}{\mathrm{snr}} \mathbf{I}\right)^{-1} \mathbf{H}_{i-1}^{*}\right]
$$

Inserting (28) into (27) yields

$$
\begin{aligned}
\rho_{i}^{\mathrm{MMSE}}= & \operatorname{snrh}_{i}^{*} \mathbf{P}_{\mathbf{H}_{i-1}}^{\perp} \mathbf{h}_{i}+ \\
& \operatorname{snh}_{i}^{*} \mathbf{H}_{i-1}\left[\left(\mathbf{H}_{i-1}^{*} \mathbf{H}_{i-1}\right)^{-1}-\left(\mathbf{H}_{i-1}^{*} \mathbf{H}_{i-1}+\frac{1}{\mathrm{snr}} \mathbf{I}\right)^{-1}\right] \mathbf{H}_{i-1}^{*} \mathbf{h}_{i} \text { (29) } \\
= & \rho_{i}^{\mathrm{ZF}}+ \\
& \operatorname{snrh}_{i}^{*} \mathbf{H}_{i-1}\left[\left(\mathbf{H}_{i-1}^{*} \mathbf{H}_{i-1}\right)^{-1}-\left(\mathbf{H}_{i-1}^{*} \mathbf{H}_{i-1}+\frac{1}{\mathrm{snr}} \mathbf{I}\right)^{-1}\right] \mathbf{H}_{i-1}^{*} \mathbf{h}_{i} \cdot(30)
\end{aligned}
$$

We reminder the reader that $\rho_{i}^{\mathrm{zF}}$ is given in (11). To obtain (30) from (29), we have used (11) and the fact that $\mathbf{h}_{i}^{*} \mathbf{P}_{\mathbf{H}_{i-1}}^{\perp} \mathbf{h}_{i}=r_{i i}^{2}$. Now we examine the second term of (30), for which we have the following result.

\section{Lemma IV.1 The random variable}

$\operatorname{snrh}_{i}^{*} \mathbf{H}_{i-1}\left[\left(\mathbf{H}_{i-1}^{*} \mathbf{H}_{i-1}\right)^{-1}-\left(\mathbf{H}_{i-1}^{*} \mathbf{H}_{i-1}+\frac{1}{\operatorname{snr}} \mathbf{I}\right)^{-1}\right] \mathbf{H}_{i-1}^{*} \mathbf{h}_{i}$ is statistically independent of $\rho_{i}^{Z F}$ and has the same distribution as

$$
\eta_{\mathrm{snr}} \triangleq \mathbf{g}_{i}^{*}\left(\mathbf{H}_{i-1}^{*} \mathbf{H}_{i-1}+\frac{1}{\mathrm{snr}} \mathbf{I}\right)^{-1} \mathbf{g}_{i}
$$

where $\mathbf{g}_{i} \sim N(\mathbf{0}, \mathbf{I})$ is an $(i-1)$-D circularly symmetric Gaussian vector and is statistically independent of $\mathbf{H}_{i-1}$.

Proof: Omitted.

Define

$$
\eta_{\infty}=\mathbf{g}_{i}^{*}\left(\mathbf{H}_{i-1}^{*} \mathbf{H}_{i-1}\right)^{-1} \mathbf{g}_{i} .
$$

It is easy to see that $\eta_{\mathrm{snr}} \rightarrow \eta_{\infty}$, w.p.1 (with probability one) as snr $\rightarrow \infty$. Hence at high SNR region, we can approximate $\rho_{i}^{\mathrm{MMSE}}$ as

$$
\rho_{i}^{\mathrm{MMSE}} \approx \rho_{i}^{\mathrm{ZF}}+\eta_{\infty}
$$

It can be shown that

$$
\frac{m-i+2}{i-1} \eta_{\infty} \sim \mathcal{F}_{2(i-1), 2(m-i+2)}
$$

or

$$
f_{\eta_{\infty}}(x)=\frac{m !}{(i-2) !(m-i+1) !} \frac{x^{i-2}}{(1+x)^{m+1}}, \quad x>0
$$

which is independent of snr and $\rho_{i}^{\mathrm{zF}}$ as proven in Lemma IV.1.

Intuitively, $\eta_{\infty}$ represents the power of the signal component that is "hidden" in the range space of $\mathbf{H}_{i-1}$ and is recovered by the MMSE estimator. In contrast, the ZF estimator nulls out of that signal component completely.

The cumulative density function (CDF) of $\eta_{\infty}$ and empirical cumulative density function (ECDF) of $\eta_{\mathrm{snr}}$, for $m=n=$ $i=4$, is given in Figure 1. The ECDF line is based on $10^{4}$ randomly generated $\eta_{\text {snr }}$ and is plotted using the Matlab function ECDF. We see that for the approximation of $\eta_{\mathrm{snr}}$ by $\eta_{\infty}$ is very accurate for a reasonablely high SNR (e.g., snr $=20$ $\mathrm{dB})$.

Using the typical error event analysis technique, we get the diversity gain of the $i$ th layer using MMSE-V-BLAST as follows

$$
d_{i}^{\mathrm{MMSE}}=-\lim _{\mathrm{snr} \rightarrow \infty} \frac{\log P\left(\rho_{i}^{\mathrm{zF}}+\eta_{\infty}<1\right)}{\log \mathrm{snr}} .
$$

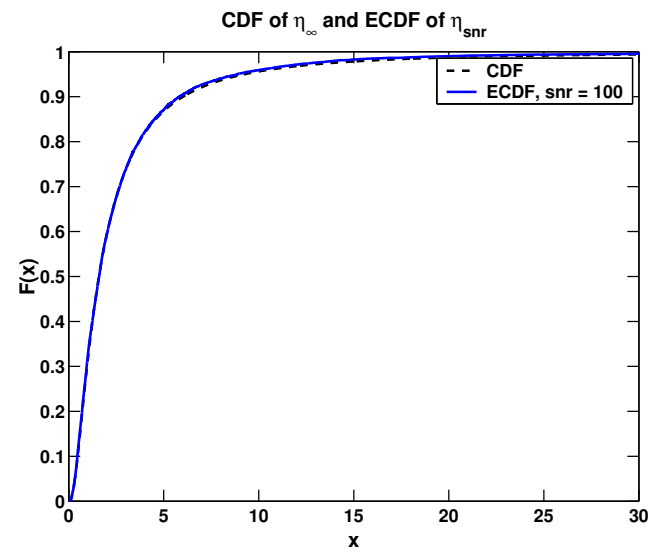

Figure 1: Cumulative distribution function (CDF) of $\eta_{\infty}$ and empirical cumulative distribution function (ECDF)of $\eta_{\mathrm{snr}}$ based on $10^{4}$ randomly generated variables. $m=n=i=4$, $\mathrm{snr}=100(20 \mathrm{~dB})$

Although the probability of the typical error event of MMSEV-BLAST is smaller than its ZF counterpart, we show that the effect of $\eta_{\infty}$ on the diversity gain will diminish as snr $\rightarrow \infty$ since $\eta_{\infty}$ is independent of snr. Hence we give the following proposition.

Theorem IV.2 The diversity gain of the nth layer using the MMSE-V-BLAST scheme is

$$
d_{n, M M S E}=m-n+1 \text {. }
$$

Moreover, applying optimal ordering does not improve the diversity gain.

Proof: Similar to ZF-V-BLAST, we apply the typical error event analysis technique to derive the diversity gain of MMSE-V-BLAST

$$
d_{n, \mathrm{MMSE}}=-\lim _{\mathrm{snr} \rightarrow \infty} \frac{\log P\left(r_{n n}^{2} \mathrm{snr}+\eta_{\infty}<1\right)}{\log \mathrm{snr}}
$$

It is easy to see that

$$
\begin{aligned}
\log P & \left(r_{n n}^{2} \mathrm{snr}+\eta_{\infty}<1\right) \\
>\quad & \log P\left(r_{n n}^{2}<\mathrm{snr}^{-1} / 2, i=1, \ldots, n\right) \\
& +\log P\left(\eta_{\infty}<1 / 2\right)
\end{aligned}
$$

In the right hand side of (38), the second term is a strictly positive number and is not related to snr, and the first term is proportional to $\mathrm{snr}^{-(m-n+1)}$ as it can be readily checked from the proof of Theorem III.1. Hence $d_{i, \text { MMSE }} \leq m-n+1$. However, $d_{i, \mathrm{MMSE}} \geq d_{i, \mathrm{ZF}}=m-n+1$. Hence $d_{i, \mathrm{MMSE}}=$ $m-n+1$. The proof of the diversity gain of ordered MMSE-VBLAST is rather complicated, which we omit here for limited space.

However, diversity analysis is not sufficient to shed light on the remarkable BER performance gap between the two versions of V-BLAST. We need to compare the BER performance of MMSE-V-BLAST and ZF-V-BLAST more closely.

Again, we focus on studying the $n$th layer. We consider the case where noncoherent binary orthogonal FSK (BFSK) is 
used. Denote $\rho$ as the post-processing SNR. Then the BER using BFSK is [4]

$$
P_{e}(\rho)=\frac{1}{2} e^{-\rho / 2}
$$

The BER performance of ZF-V-BLAST can be evaluated as

$$
P_{e, \text { BFSK }}^{\mathrm{ZF}}=\int_{0}^{\infty} \frac{1}{2(m-n) !} e^{-\frac{x \mathrm{snn}}{2}} x^{m-n} e^{-x} d x
$$

After some straightforward calculations, we obtain

$$
P_{e, \mathrm{BFSK}}^{\mathrm{ZF}}=\frac{1}{2}\left(\frac{1}{1+\frac{1}{2} \mathrm{snr}}\right)^{m-n+1} .
$$

Similarly, the BER of MMSE-V-BLAST can be calculated as

$$
\begin{aligned}
P_{e, \mathrm{BFSK}}^{\mathrm{MMSE}} & =E_{\eta}\left[\int_{0}^{\infty} \frac{1}{2(m-n) !} e^{-\frac{x \mathrm{ssn} r+\eta}{2}} x^{m-n} e^{-x} d x\right] \\
& =P_{e, \mathrm{BFSK}}^{\mathrm{ZF}} E_{\eta}\left[e^{-\eta / 2}\right] \\
& \triangleq \mu(m, n) P_{e, \mathrm{BFSK}}^{\mathrm{ZF}},
\end{aligned}
$$

where

$$
\mu(m, n)=\int_{0}^{\infty} e^{-\eta / 2} \frac{m !}{(n-2) !(m-n+1) !} \frac{\eta^{n-2}}{(1+\eta)^{m+1}} d \eta
$$

is the BER ratio between $P_{e, \mathrm{BFSK}}^{\mathrm{MMSE}}$ and $P_{e, \mathrm{BFSK}}^{\mathrm{ZF}}$. Clearly, $0<\mu<$ 1. At high SNR, we can approximate (41) as

$$
P_{e, \mathrm{BFSK}}^{\mathrm{ZF}} \approx \frac{2^{m-n}}{\mathrm{snr}^{m-n+1}}
$$

Hence the BER performance gain $\mu$ is associated with the post-processing SNR gain as

$$
G(m, n)=-\frac{10}{m-n+1} \log _{10} \mu(m, n) .
$$

An analytical expression for $\mu(m, n)$ is difficult. Numerical computations show that $\mu(m, n)$ can be quite small. Some typical cases are presented as follows.

\begin{tabular}{l|c|c|c}
\hline$m$ & $n$ & $\mu(m, n)$ & $G(m, n)(\mathrm{dB})$ \\
\hline 4 & 3 & 0.6779 & 0.84 \\
\hline 4 & 4 & 0.4372 & 3.59 \\
\hline 6 & 6 & 0.2835 & 5.49 \\
\hline 10 & 10 & 0.1362 & 8.66 \\
\hline
\end{tabular}

Hence at the high SNR region, MMSE-V-BLAST can still have considerablely smaller BER than ZF-V-BLAST. Figure 2 presents the comparative result on the BER performances of the $n$th layer using MMSE-V-BLAST and ZF-V-BLAST. The upper line (dash-dot line) represents $P_{e, \mathrm{BFSK}}^{\mathrm{ZF}}$. The dashed line represents $P_{e, \text { BFSK }}^{\mathrm{ZF}}$ given in (42), and the solid line is the true BER performance of MMSE-V-BLAST based on the postprocessing SNR given in (26) and the error probability in (39). The true BER is obtained via $10^{4}$ Monte-Carlo trials. We see that $P_{e, \text { BFSK }}^{\mathrm{ZF}}$ closely fits the true BER performance of MMSEV-BLAST especially for high SNR, which is because $\eta_{\infty}$ approximates $\eta_{\text {snr }}$ very well at high SNR.

To conclude this section, we remark that the $n$th layer of VBLAST, without using optimal ordering, has the same statistic of the conventional linear equalizers, including the channel inverse equalizer and the linear MMSE (LMMSE) equalizer. Hence our analysis also captures the performance difference between the two linear equalizers.

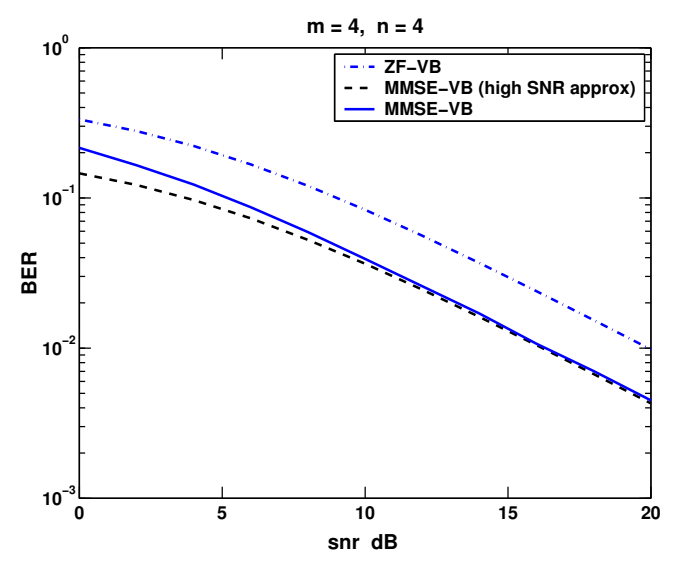

Figure 2: BER performance of the $n$th layer using ZF-VBLAST and MMSE-V-BLAST, where $m=n=4$.

\section{CONCLUSIONS}

In this paper, we present an asymptotic analysis of the VBLAST scheme at high SNR region. Both the ZF-V-BLAST and MMSE-V-BLAST are analyzed with respect to their diversity gains and BER performances. We show that for both ZF-V-BLAST and MMSE-V-BLAST, applying optimal ordering does not improve the diversity gain. We show that the gap between the output SNRs of MMSE and ZF estimators has a scaled $\mathcal{F}$ distribution asymptotically for high SNR. We also quantify the remarkable BER performance advantage of the MMSE-V-BLAST over the ZF-V-BLAST for high SNR.

\section{REFERENCES}

[1] G. J. Foschini, G. D. Golden, R. A. Valenzuela, and P. W. Wolniansky, "Simplified processing for high spectral efficiency wireless communication employing multiple-element arrays," Wireless Personal Communications, vol. 6, pp. 311-335, March 1999.

[2] G. Ginis and J. M. Cioffi, "On the relationship between V-BLAST and the GDFE," IEEE Communications letters, vol. 5, pp. 364-366, September 2001.

[3] M. Varanasi and T. Guess, "Optimum decision feedback multiuser equalization with successive decoding achieves the total capacity of the Gaussian multiple-access channel," Conference Record of the ThirtyFirst Asilomar Conference on Signals, Systems and Computers, vol. 2, pp. 1405 - 1409, Nov 2-5 1997.

[4] S. Loyka and F. Gagon, "Performance analysis of the V-BLAST algorithm: an analytical approach," IEEE Transactions on Wireless Communications, vol. 3, pp. 1326-1337, July 2004.

[5] L. Zheng and D. Tse, "Diversity and multiplexing: A fundamental tradeoff in multiple-antenna channels," IEEE Transactions on Information Theory, vol. 49, pp. 1073-1096, May 2003.

[6] D. Tse and P. Viswanath, Fundamentals of Wireless Communications. Available: http://inst.eecs.berkeley.edu/ ee224b/sp04/\#Course Notes, 2004.

[7] A. M. Tulino and S. Verdu, Random Matrix Theory and Wireless Communications. Hanover, MA 02339, USA: now Publishers Inc., 2004.

[8] R. A. Horn and C. R. Johnson, Matrix Analysis. Cambridge: Cambridge University Press, 1985. 